\title{
A Review on Object Tracking Across Real-World Multi Camera Environment
}

\author{
Rino Cherian \\ Research Scholar \\ KVJCE, Sullia
}

\author{
Jothimani K., PhD \\ Professor \\ KVJCE, Sullia
}

\author{
Reeja S.R., PhD \\ Associate Professor \\ DSU-SoE[39-51]
}

\begin{abstract}
Object identification, tracking and monitoring are significant and testing assignments in numerous computer vision applications, for example, surveillance, vehicle navigation and self-governing robot navigation. Video reconnaissance in a powerful environment, particularly for people and vehicles, is one of the momentum testing research points. It is a key innovation to battle against illegal activity, offence, public security and for effective administration of traffic. The work includes planning of the proficient video observation framework in complex conditions. In video observation, recognition of moving items from a video is significant for object discovery, target tracking, and behavior understanding. Recognition of moving items in video transfers is the primary significant advance of data and background subtraction is a famous methodology for frontal segmentation. In this paper, after surveying ongoing advances of online article, we do enormous scope tries different things with different assessment standards to see how these algorithms perform. By breaking down quantitative outcomes, we distinguish viable methodologies for powerful following and give potential future examination bearings in this field.
\end{abstract}

\section{Keywords}

Object Tracking

\section{INTRODUCTION}

These days, utility of video surveillance turns into a typical truth in numerous fields. Among these reconnaissance frameworks, products cameras are utilized to improve coverage and accuracy in the reviewed territory. Because of the immense measure of information created by these video reconnaissance frameworks; the requirement for programmed investigation procedures that might be utilized to abuse these information turns out to be more imperative to consider the conduct of individuals without requiring numerous human administrators to examine the caught recordings. Individuals' tracking in video transfer is the essential advance for insightful observation applications in various fields, for example, the programmed social investigation of individuals, and the security reconnaissance frameworks. Consequently, numerous investigates had been led, and an assortment of approaches had been utilized to take care of this issue. The issue of individuals tracking is tended to in two principle thematic. The mono-camera design in which just information caught from a solitary camera are prepared during tracking cycle, and the multi-camera arrangement in which the correspondences between tracking people among the entire framework are thought of. Both mono and multi-camera tracking of individuals are characterized as the capacity of naturally recording the directions of tracking people in the overviewed scene. The principle contrast between these arrangements live in the way that in multi-camera frameworks the correspondences between the monitoring people in various cameras are thought of while in the mono-camera frameworks these correspondences are simply overlooked. This tracking might be accomplished either continuously which is known as the web based tracking or from a recorded video which is known as the disconnected monitoring. [9] The issue of individuals tracking in a multi-camera framework is an expansion of the issue of individuals tracking in mono-camera framework. Accordingly, a large portion of the calculations which are utilized in multi-camera monitoring depend on the notable calculations of mono-camera monitoring, for example, the movement recognition, object displaying and include extraction strategies. The utilization of multi-camera has numerous points of interest over the utilization of a solitary camera. Among these preferences, we may make reference to the decrease of mistakes that are brought about by impediments in packed climate and disappointments that may influence any sensor and the capacity of checking enormous regions. In any case, in the other hand, individuals tracking in multi-camera framework is exceptionally testing, on the grounds that the tracking cycle safeguard to meld data originating from the various sensors. In this unique circumstance, numerous investigates had been directed over the most recent couple of years to take care of this issue [9, $10]$.

\section{LITERATURE SURVEY}

The author [1] disclosed to identify and follow items continuously, we zeroed in on the concurrent pre-preparing of recordings from numerous cameras for vigorous item recognition. In the wake of synchronizing different recordings, superfluous edges with generally little data are taken out dependent on change identification. An article score is estimated on a casing by-outline premise to choose the most critical edges. Next, the objective items are recognized and followed in the chose outlines. At long last, the buying conduct of the distinguished item is perceived dependent on the following data. These cycles were utilized to plan a start to finish acknowledgment structure. The commitment of this paper is huge in that by upgrading the current profound neural organizations a continuous coordinated framework for a functional application was effectively acknowledged with no bottleneck from multi-camera contributions to conclusive article acknowledgment measure. Moreover, the proposed object identification network shows practically identical execution with the cutting edge techniques. We performed escalated trials to assess unadulterated item identification execution just as to assess different buy/bring situations back. For instance, for a fundamental buy/return situation, the proposed framework accomplished about $92 \%$ or more precision, which can be the real degree of commercialization.

The author [2] says that continuous article location and following is an immense, lively yet uncertain region of PC vision. Programmed object identification and following are valuable in observation, global positioning frameworks 
utilized in security, versatile robots, clinical treatment, driver help frameworks, and examination of sports. Calculations proposed in existing writing use shading division, edge following, shape identification for recognition, and following of an article. The difficulties, for example, following in powerful climate and troublesome following of various articles in numerous camera climate and costly calculation confine the execution of these frameworks for tackling genuine issues. This spurs us to build up a framework that is effective progressively object discovery and following. In this paper, creators build up the continuous article location and global positioning framework utilizing speed control. Test results demonstrate its adequacy in recognition and following of straightforward just as perplexing items in both basic and complex foundations. The framework is powerful in recognizing and following the co-event of two articles. It obviously shows the effect of shading strength or shape predominance, self-shadow, and picture of an article in a mirror.

In this paper [3] image based monitoring of individual can give rich information to support achievements in organic and clinical exploration, yet hardly any current techniques stretch out to following unconstrained regular conduct in the field. We have built up a visual global positioning framework for animals shot with a uninhibitedly moving hand-held or droneworked camera in their indigenous habitat. This adventures a worldwide deduction strategy for identifying movement of a creature against a jumbled foundation. Directions are then produced by a novel video key-outline determination conspire in blend with a mathematically obliged image sewing calculation, bringing about a two-dimensional scene image of the climate on which the thick creature way is shown. By presenting a negligible and conceivable arrangement of imperatives in regards to the camera direction and development, we exhibit that both per-outline creature positions and in general directions can be removed with sensible exactness, for a scope of various animals, conditions and imaging modalities. This strategy requires just a solitary un-calibrated camera, doesn't need checking or preparing information to distinguish the creature, and shows up of the objective or foundation. Specifically it can recognize targets involving less than 20 pixels in the image, and manage helpless differentiation, exceptionally powerful lighting and continuous impediment. This calculation creates exceptionally useful subjective directions installed in a scene of the climate. The outcomes are as yet dependent upon rotational float and extra scaling schedules would be expected to acquire total true facilitates. It in any case gives an adaptable and simple toutilize framework to acquire rich information on normal animal conduct in the field. In this paper [4] the author says that a computerized moving item recognition and examination accepts an incredible centrality in video observation. This article presents a complete study on the procedures of item moving identification for video observation. In this paper, eight techniques for object location in video transfers are executed and assessed exactly on five quality boundaries for distinguishing the proficiency and viability of these strategies. For target evaluations of these strategies, a standard dataset "CDnet2012" is utilized which comprises of six distinctive thorough situations. Taking everything into account, an endeavor has been made to distinguish the best technique for various situations, employable progressively video reconnaissance.

In this paper [5] Object tracking in RGB-Thermal (RGB-T) recordings is progressively utilized in numerous fields because of the all-climate and the entire day working capacity of the double methodology imaging framework, just as the quick advancement of ease and scaled down infrared camera innovation. In any case, it is still exceptionally testing to viably combine double methodology data to assemble a hearty RGB-T tracker. In this paper, a RGB-T object tracking calculation dependent on a modular mindful consideration organization and serious learning (MaCNet) is proposed, which incorporates an element extraction organization, modular mindful consideration organization, and arrangement organization. The element extraction network receives the type of a two-stream organization to extricate highlights from every methodology picture. The modular mindful consideration network incorporates the first information, builds up a consideration model that describes the significance of various element layers, and afterward controls the element combination to improve the data association between modalities. The order network develops a methodology proud misfortune work through three parallel binary classifiers following up on the RGB branch, the thermal infrared branch, and the combination branch, individually. Guided by the training technique of competitive learning, the whole network is tweaked toward the ideal combination of the double modalities. Broad experiments on a few openly accessible RGB-T datasets show that our tracker has better execution looked at than other most recent RGB-T and RGB tracking methodologies.

This paper [6] portrays another technique for the location of moving items from moving camera image groupings utilizing an inertial measurement unit (IMU) sensor. Movement discovery frameworks with vision sensors have become a worldwide examination subject as of late. Nonetheless, identifying moving items from a moving camera is a troublesome errand as a result of ego motion. In the proposed strategy, the intriguing focuses are separated by a Harris indicator, and the foundation and forefront are arranged by epi-polar geometry. In this technique, an IMU sensor is utilized to ascertain the underlying principal network. After the element point order, a change framework is gotten from coordinating foundation include focuses. image enrollment is then applied to the continuous images, and a distinction map is removed to discover the forefront district. At long last, a base jumping box is applied to check the identified moving article. The proposed technique is actualized and tried with various certifiable driving recordings, which show that it beats the past work.

For table-tennis robots, ball tracking and direction forecast are the most significant advancements. A few techniques were created in past exploration endeavors, and they can be separated into two classes: physical models and AI. This examination subsequently utilized AI to become familiar with the flight directions of ping-pong balls, which comprise of two explanatory directions: one start at the serving point and finishing at the arrival point on the table, and the other start at the arrival point and finishing at the striking purpose of the robot. We [7] set up two fake neural organizations to get familiar with these two directions. We directed a training test utilizing 200 genuine directions as preparing information. The mean blunders of the proposed dual network technique and a single network model were $39.6 \mathrm{~mm}$ and $42.9 \mathrm{~mm}$, respectively. The outcomes demonstrate that the expectation execution of the proposed dual network technique is superior to that of the single-network approach. We likewise utilized the physical model to produce 330 directions for training and the test results show that the prepared model made a progress pace of $97 \%$ out of 30 endeavors, which was higher than the achievement pace of $70 \%$ acquired by the physical model. A 
physical investigation introduced a mean mistake and standard deviation of $36.6 \mathrm{~mm}$ and $18.8 \mathrm{~mm}$, individually. The outcomes likewise show that even without the time stamps, the proposed strategy keeps up its expectation execution with the extra points of interest of $15 \%$ less boundaries in the general network and 54\% shorter training time.

This paper [8] presents a real time 3D object identifier dependent on LiDAR based Simultaneous Localization and Mapping (LiDAR-SLAM). The 3D point mists procured by portable LiDAR frameworks, inside the environment, are typically profoundly spars, unpredictably disseminated, and regularly contain impediment and basic equivocalness. Existing 3D object recognition techniques dependent on Convolutional Neural Networks (CNNs) depend intensely on both the strength of the 3D features and a lot of labelling. A key test is effective discovery of 3D objects in point clouds of huge scope building conditions without pre-training the 3D CNN model. To extend image based article identification results and LiDAR-SLAM results onto a 3D likelihood map, we consolidate visual and run data into a frustum-based probabilistic structure. Accordingly, we tackle the sparse and noise issue in LiDAR-SLAM information, in which any point cloud descriptor can scarcely be applied. The 3D object identification results, gotten utilizing both knapsack LiDAR dataset and the notable KITTI Vision Benchmark Suite, show that our strategy beats the cutting edge strategies for object confinement and jumping box assessment.

Various techniques for individuals following in multi-camera framework is clarified in this paper $[11-21]$. The Fig. 1 shows the embraced classification for multi-camera monotoring techniques. In centralized methodology there is sure essential methodology which is outlined in fig.2 and Table 1 shows Centralized methodologies with different features. The Fig. 3 shows essential distributed methodology and Table 2 shows distributed approaches with different features [22-37].

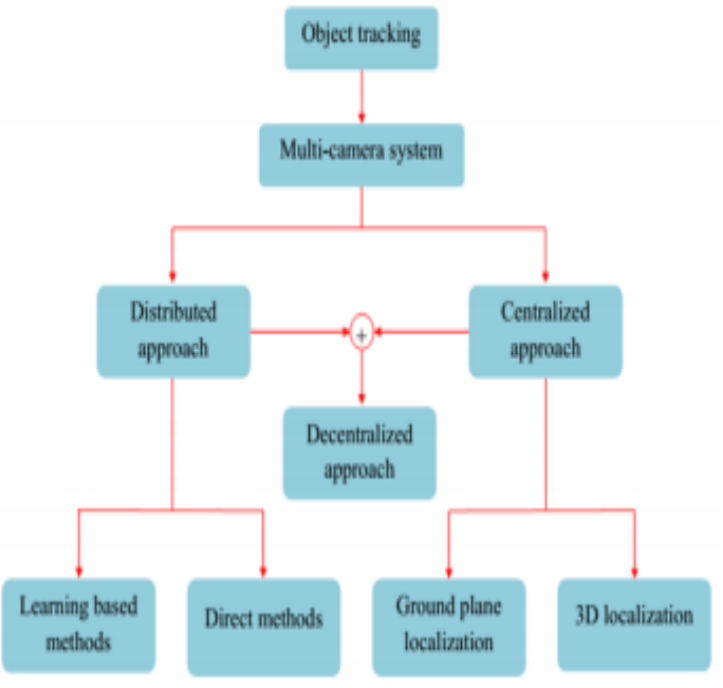

Fig. 1 Adopted classification for multi-camera tracking methods

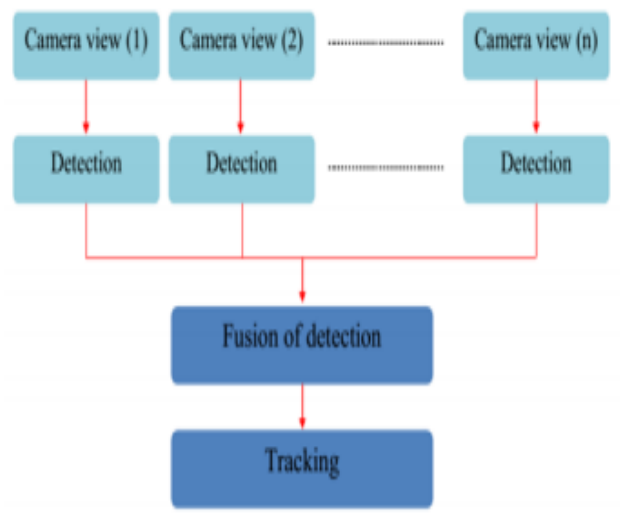

Fig. 2 Centralized approach

Table 1 Centralized approaches

\begin{tabular}{llll}
\hline Reference & Feature & Association method & Calibration \\
\hline $\begin{array}{l}\text { Kim et al } \\
\text { Du et al }\end{array}$ & Binary map & $\begin{array}{l}\text { intersection of the central } \\
\text { vertical axis }\end{array}$ & Yes \\
\hline $\begin{array}{l}\text { Feuret et al } \\
\text { Huang et al }\end{array}$ & Binary map & Occupancy map & Yes \\
\hline $\begin{array}{l}\text { Santos et al } \\
\text { Khan et al }\end{array}$ & Binary map & Homgraphy constraints & No \\
\hline $\begin{array}{l}\text { Liem et al } \\
\text { Guan et al } \\
\text { Arsic et al }\end{array}$ & Color & 3D reconstruction & Yes \\
\hline Chen et al & Tracklets & & \\
\hline Krocger et al & Color & global graph optimization & No \\
\hline Yao et al & Color appearance & SFM & No \\
\hline $\begin{array}{l}\text { Hofmann et al } \\
\text { Leal-Taixe et al }\end{array}$ & Color & 3D model & Yes \\
\hline Bredereck et al & HSV color and positions & Deformable part model, 3D & Yes \\
\hline $\begin{array}{l}\text { Jiaung et al } \\
\text { Baltein et al }\end{array}$ & Color appearance & $\begin{array}{l}\text { 3D positions } \\
\text { distances }\end{array}$ & Yes \\
\hline Wen et al & appearance, motion continuity & 3D positions & Yes \\
\hline Du et al & Color appearance & Probabilistic algorithm & No \\
\hline Zhang and Cheng & haar-like features, HOG, and LBP & combining views & Yes \\
\hline & & & Yes \\
\hline
\end{tabular}

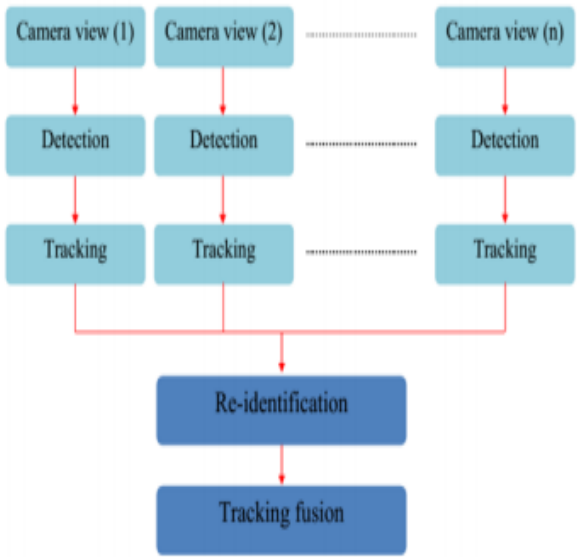

Fig. 3 Distributed approach 
Table 2 Distributed approaches

\begin{tabular}{|c|c|c|c|}
\hline Reference & Feature & Association method & Calibration \\
\hline $\begin{array}{l}\text { Back et al } \\
\text { Kuo et al }\end{array}$ & $\begin{array}{l}\text { Haar-like } \\
\text { color histogram, } \\
\text { covariance matrix, } \\
\text { HOG }\end{array}$ & Adaboost & 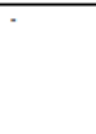 \\
\hline $\begin{array}{l}\text { Avraham et al } \\
\text { Kenk et al }\end{array}$ & $\begin{array}{l}\text { HOG } \\
\text { Color histogram }\end{array}$ & SVM & $\cdot$ \\
\hline Nakajama et al & HOG & Multi-class SVM & $\cdot$ \\
\hline Prosser et al & Color, texture & RankSVM & $\cdot$ \\
\hline Loy et al & Color & Laplacian graph & - \\
\hline Bazzani et al & histogram plus epitome & K-NN & - \\
\hline Bak et al & Covariance descriptor & SVM & $\cdot$ \\
\hline Javed et al & Color & Epipolar geometry & Yes \\
\hline Aziz et al & $\begin{array}{l}\text { SIFT, SURF, } \\
\text { SpinImage }\end{array}$ & 2D model, $\mathrm{k}$-NN & Yes \\
\hline $\begin{array}{l}\text { Cheng et al } \\
\text { Wang et al }\end{array}$ & Color, texture & $\cdot$ & $\cdot$ \\
\hline Cong et al & Color & 2D model, $\mathrm{k}-\mathrm{NN}$ & $\cdot$ \\
\hline Chen et al & Color spectrum & Tracklet association & $\cdot$ \\
\hline Hamdoun et al & SURF & Voting algorithm, K-NN & $\cdot$ \\
\hline Farenzena et al & $\begin{array}{l}\text { Several appearance } \\
\text { features }\end{array}$ & $\begin{array}{l}\text { Asymmetric and } \\
\text { symmetric axis of } \\
\text { human body model }\end{array}$ & $\cdot$ \\
\hline Mazzon and Cavallaro & Position and velocity & Social force model & - \\
\hline Bouma et al & Color and spatial information & Histogram intersection & $\cdot$ \\
\hline Nie et al & HSV, HOG, spatial information & Graph matching & $\cdot$ \\
\hline Brendel et al & $\begin{array}{l}\text { Position, size, color } \\
\text { histograms, intensity } \\
\text { gradient, motion }\end{array}$ & MWIS algorithm & $\cdot$ \\
\hline Lietal & Color & $\cdot$ & $\cdot$ \\
\hline Colombo et al & $\begin{array}{l}\text { spatio-temporal properties, } \\
\text { Color }\end{array}$ & Distance measure & Yes \\
\hline
\end{tabular}

As depicted in table 3, there are no discriminative rules that permit choosing whether the centralized methodology is superior to the distributed one or the other way around. Both the concentrated and the distributed methodologies have a few advantages and downsides. In this way, the decision of adopted approach depends, by and large, in the details of the planned application as appeared in the table 3 .
Table 3 Summary of usage and comparison between the centralized and the distributed approaches

\begin{tabular}{|c|c|c|}
\hline & Centralized approach & Distributed approach \\
\hline है & $\begin{array}{l}\text { - Monitoring of small areas. } \\
\text { - Cameras with overlapping views. } \\
\text { - Crowded areas. }\end{array}$ & $\begin{array}{l}\text { - Monitoring of large areas. } \\
\text { - Non-overlapping cameras views. }\end{array}$ \\
\hline ֻू & $\begin{array}{l}\text { - Exploit the multi-views of the scene. } \\
\text { - Well handling of occlusions. } \\
\text { - Reduced effect of noisy observations } \\
\text { and failure in a single sensor. }\end{array}$ & $\begin{array}{l}\text { - No need for synchronization. } \\
\text { - Accurate Geometric relationship be- } \\
\text { tween cameras is not necessary for } \\
\text { tracking. } \\
\text { - Just few cameras are needed. } \\
\text { - Suitable for online applications. }\end{array}$ \\
\hline 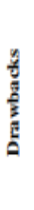 & $\begin{array}{l}\text { - Synchronization of cameras is needed. } \\
\text { - Accurate Geometric relationship be- } \\
\text { tween cameras is necessary for track- } \\
\text { ing. } \\
\text { - Large number of cameras is needed for } \\
\text { a small area. } \\
\text { - Not very suitable for online applica- } \\
\text { tions. }\end{array}$ & $\begin{array}{l}\text { - Use just a single view even in the avail- } \\
\text { ability of multi-views. } \\
\text { - Problem in case of severe occlusions. } \\
\text { - Noisy observations or failure in any } \\
\text { sensor affects the whole tracking sys- } \\
\text { tem. }\end{array}$ \\
\hline 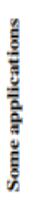 & \multicolumn{2}{|c|}{$\begin{array}{l}\text { - Intelligent surveillance systems. } \\
\text { - Urban traffic control. } \\
\text { - Monitoring of people in public places. } \\
\text { - Pedestrian counting. } \\
\text { - Behavioral analysis of peoples. } \\
\text { - Sport analysis. } \\
\text { - Statistical studies for commercial pur- } \\
\text { poses. }\end{array}$} \\
\hline
\end{tabular}

\section{CONCLUSION}

We introduced an outline of the current techniques for individuals' following in multi-camera frameworks. We had arranged these strategies into two fundamental classes the centralized and the distributed approaches depending in information combination between various cameras. The centralized methodologies are joining information from various sensors so as to recuperate the ground plane inhabitance map or the 3D directions of the followed people though the distributed approaches are consolidating only the directions got by free following in every camera to acquire the worldwide direction of the monitored person. In every one of these classes an assortment of techniques were proposed. Hence, we summed up the most known strategy for every one of these classes to get a diagram that notice the majority of the pre-owned methodologies without referencing all the proposed techniques

\section{REFERENCES}

[1] Kim, Dae Ha, et al. "Real-time purchase behavior recognition system based on deep learning-based object detection and tracking for an unmanned product cabinet", International Journal of Expert Systems with Applications, Elsevier, Volume 143, 1 April 2020, 11306

[2] Rani, Geeta, and Anita Jindal. "Real-Time Object Detection and Tracking Using Velocity Control," Smart Systems and IoT: Innovations in Computing, Springer, Singapore, 2020. 767-778.

[3] Haalck, Lars, et al. "Towards image-based animal tracking in natural environments using a freely moving camera." Journal of neuroscience methods Elsevier, Volume 330 (2020): 108455.

[4] Singh, Surender, et al. "Object Motion Detection Methods for Real-Time Video Surveillance: A Survey with Empirical Evaluation." Smart Systems and IoT: Innovations in Computing, Springer, Singapore, 2020. 663-679. 
[5] Zhang, Hui, et al. "Object Tracking in RGB-T Videos Using Modal-Aware Attention Network and Competitive Learning." Sensors 20.2 (2020): 393.

[6] Jung, Sukwoo, et al. "Moving object detection from moving camera image sequences using an inertial measurement unit sensor." Applied Sciences 10.1 (2020): 268

[7] Lin, Hsien-I., Zhangguo Yu, and Yi-Chen Huang. "Ball Tracking and Trajectory Prediction for Table-Tennis Robots", Sensors 20.2 (2020): 333.

[8] Gong, Zheng, et al. "A Frustum-based probabilistic framework for 3D object detection by fusion of LiDAR and camera data." ISPRS Journal of Photogrammetry and Remote Sensing . Elsevier, Volume 159 (2020): 90-100.

[9] Evans M, Osborne CJ, Ferryman JM (2013) Multicamera object detection and tracking with object size estimation. In: AVSS, IEEE, pp 177-182

[10] Youlu W (2013) Distributed multi-object tracking with multi-camera systems composed of overlapping and nonoverlapping cameras, $\mathrm{PhD}$ thesis, University of NebraskaLincoln.

[11] Iguernaissi, Rabah, et al. "People tracking in multicamera systems: a review." Multimedia Tools and Applications, springer, 78.8 (2019): 10773-10793.

[12] del Blanco CR, Mohedano R, Garca NN, Salgado L, Jaureguizar F (2008) Color-based 3d particle filtering for robust tracking in heterogeneous environments. In: ICDSC, IEEE, pp 1-10

[13] Fleuret F, Berclaz J, Lengagne R, Fua P (2008) Multicamera people tracking with a probabilistic occupancy map. Pattern Analysis and Machine Intelligence, IEEE Transactions on 30(2):267-282

[14] Huang C, Wang SJ (2012) A bayesian hierarchical framework for multitarget labeling and correspondence with ghost suppression over multicamera surveillance system. IEEE $\mathrm{T}$ Automation Science and Engineering 9(1):16-30

[15] Khan SM, Shah M (2006) A multiview approach to tracking people in crowded scenes using a planar homography constraint. In: Leonardis A, Bischof H, Pinz A (eds) ECCV (4), Springer, Lecture Notes in Computer Science, vol 3954, pp 133-146

[16] Kim K, Davis LS (2006) Multi-camera tracking and segmentation of occluded people on ground plane using search- guided particle filtering. In: In ECCV, pp 98-109

[17] Santos TT, Morimoto CH (2011) Multiple camera people detection and tracking using support integration. Pattern Recogn Lett 32(1):47-55, DOI 10.1016/j.patrec.2010.05.016, http://dx.doi.org/10.1016/j.patrec.2010.05.016

[18] Khan SM, Shah M (2006) A multiview approach to tracking people in crowded scenes using a planar homography constraint. In: Leonardis A, Bischof H, Pinz A (eds) ECCV (4), Springer, Lecture Notes in Computer Science, vol 3954, pp 133-146

[19] Liem MC, Gavrila DM (2014) Joint multi-person detection and tracking from overlapping cameras. Computer Vision and Image Understanding 128:36-50
[20] Chen W, Cao L, Chen X, Huang K (2016) An equalized global graph model-based approach for multi-camera object tracking. IEEE Transactions on Circuits and Systems for Video Technology

[21] Arsic D, Hristov E, Lehment NH, Hrnler B, Schuller B, Rigoll G (2008) Applying multi layer homography for multi camera person tracking. In: ICDSC, IEEE, pp 1-9

[22] Kuo CH, Huang C, Nevatia R (2010) Multi-target tracking by on-line learned discriminative appearance models. In: CVPR, IEEE, pp 685-692

[23] Zheng WS, Gong S, Xiang T (2013) Reidentification by relative distance comparison. IEEE Trans Pattern Anal Mach Intell 35(3):653-668

[24] Nie W, Liu A, Su Y, Luan H, Yang Z, Cao L, Ji R (2014) Single/cross-camera multipleperson tracking by graph matching. Neurocomputing 139:220-232

[25] Lin Z, Davis LS (2008) Learning pairwise dissimilarity profiles for appearance recognition in visual surveillance. In: Bebis G, Boyle RD, Parvin B, Koracin D, Remagnino P, Porikli FM, Peters J, Klosowski JT, Arns LL, Chun YK, Rhyne TM, Monroe L (eds) ISVC (1), Springer, Lecture Notes in Computer Science, vol 5358, pp 23-34

[26] Mazzon R, Cavallaro A (2013) Multi-camera tracking using a multi-goal social force model. Neurocomputing 100:41-50 61.

[27] [27] Mazzon R, Tahir SF, Cavallaro A (2012) Person reidentification in crowd. Pattern Recognition Letters 33(14):1828-1837 62.

[28] [28]Medeiros H, Park J, Kak A (2008) Distributed object tracking using a cluster-based kalman filter in wireless camera networks. Selected Topics in Signal Processing, IEEE Journal of 2(4):448-463, DOI 10.1109/JSTSP.2008.2001310

[29] Javed O, Shafique K, Rasheed Z, Shah M (2008) Modeling inter-camera space-time and appearance relationships for tracking across non-overlapping views. Computer Vision

[30] Hamdoun O, Moutarde F, Stanciulescu B, Steux B (2008) Person re-identification in multi-camera system by signature based on interest point descriptors collected on short video sequences. In: ICDSC, IEEE, pp 1-6

[31] Bouma H, Borsboom S, den Hollander RJ, Landsmeer SH, Worring M (2012) Reidentification of persons in multi-camera surveillance under varying viewpoints and illumination. In: SPIE Defense, Security, and Sensing, International Society for Optics and Photonics, pp $83,590 \mathrm{Q}-83,590 \mathrm{Q}$

[32] Farenzena M, Bazzani L, Perina A, Murino V, Cristani M (2010) Person reidentification by symmetry-driven accumulation of local features. In: CVPR, IEEE, pp 2360-2367

[33] Chen W, Cao L, Chen X, Huang K (2014) A novel solution for multi-camera object tracking. In: 2014 IEEE International Conference on Image Processing (ICIP), IEEE, pp 2329-2333

[34] [34] Chen W, Cao L, Chen X, Huang K (2016) An equalized global graph model-based approach for multicamera object tracking. IEEE Transactions on Circuits and Systems for Video Technology 22. 
[35] Chen Y, Zhu X, Gong S, et al (2018) Person reidentification by deep learning multiscale representations 24. Cong DNT, Khoudour L, Achard C, Meurie C, Lezoray O (2010) People reidentification by spectral classification of silhouettes. Signal Processing 90(8):2362- 2374

[36] Kenk VS, Kovaci` c S, Kristan M, Hajdinjak M, Per ` s J, et al (2015) Visual re- " identification across large, distributed camera networks. Image and Vision Computing 34:11-26

[37] Nakajima C, Pontil M, Heisele B, Poggio T (2003) Fullbody person recognition system. Pattern Recognition 36(9):1997-2006

[38] S. R. Reeja and N. P. Kavya, "Real time video denoising," 2012 IEEE International Conference on Engineering Education: Innovative Practices and Future Trends (AICERA), Kottayam, 2012, pp. 1-5, doi: 10.1109/AICERA.2012.6306745

[39] Reeja S.R and N P Kavya. Article: Noise Reduction in Video Sequences The State of Art and the Technique for Motion Detection. International Journal of Computer Applications 58(8):31-36, November 2012

[40] Reeja S.R and N P Kavya, Noise Reduction in Video Sequences - The State of Art and the Technique for Motion Detection, International Journal of Computer Applications (0975 - 8887) Volume 58- No.8, November 2012

[41] Dias, Norman \& Reeja. (2018). A quantitative report on the present strategies of Graphical authentication. International Journal of Computer Sciences and Engineering. 06. 64-73. 10.26438/ijcse/v6si10.6473. 12.

[42] Reeja S R, Kumar Abhishek Gaurav, Ladly Patel, Rino Cherian, (2018)"Garbage Management Using Internet of Things", International Journal of Computer Sciences and Engineering, Vol.06, Issue.10, pp.56-59, 2018. 13.

[43] Reeja S R, Venkat Durga Sriram, Tarun Reddy R, Venkatamanu, Rino Cherian,(2018) "Ultrasonic Distance Measurement", International Journal of Computer Sciences and Engineering, Vol.06, Special Issue.10, pp.42-44, 2018. 14.

[44] Kaveri Hiremath, Dr. Reeja S. R, 2017, A Survey on Self Adjusting Slots \& Dynamic Job Ordering for Mapreduce Workloads using Homogeneous and Heterogeneous Hadoop Cluster, INTERNATIONAL JOURNAL OF
ENGINEERING RESEARCH \& TECHNOLOGY (IJERT) ICPCN - 2017 (Volume 5 - Issue 19)

[45] Kavya, Reeja S. R, Dr NP. (2014), "An Approach for Noise Removal from a Sequence of Video." International Journal of Scientific \& Engineering Research, Volume 5, Issue 4, April2014, pg.1266-1270, ISSN 2229-5518

[46] Reeja, S. R. (2014) "An Automated System for Detecting Congestion in Huge Gatherings.", International Journal of Computer Applications (0975 - 8887) International Conference on Information and Communication Technologies (ICICT-2014) 17.

[47] Reeja, S. R, Rino cherian,Dr Jothimani k, "PIXEL LEVEL REVERSIBLE DATA HIDING USING TWO LEVEL ENCRYPTIONS REVIEW", International Journal of Engineering Applied Sciences and Technology, 2019 Vol. 4, Issue 5, ISSN No. 2455-2143, Pages 498-503.

[48] Dr Reeja S R, Mr. Murthuza, Mr. Rino Cherian, Dr Jothimani, "A survey and development on context-aware monitoring in healthcare with big data", International Journal of Big Data Intelligence, 2020 Vol.7 No.2, pp.97 $-109$

[49] Kiran J Waghmare, Dr Reeja S R, “A Computational Intelligence Paradigm with Human Computer Interface Learning", International Journal of Innovative Technology and Exploring Engineering (IJITEE), Volume 9, Issue 2s, Page 384-389.

[50] Dias N., Reeja S.R. (2020) An Improvement of Compelling Graphical Confirmation Plan and Cryptography for Upgrading the Information Security and Preventing Shoulder Surfing Assault. In: Arai K., Bhatia R., Kapoor S. (eds) Proceedings of the Future Technologies Conference (FTC) 2019. FTC 2019. Advances in Intelligent Systems and Computing, vol 1070. Springer, Cham

[51] Soja Rani S., Reeja S.R. (2020) A Survey on Different Approaches for Malware Detection Using Machine Learning Techniques. In: Karrupusamy P., Chen J., Shi Y. (eds) Sustainable Communication Networks and Application. ICSCN 2019. Lecture Notes on Data Engineering and Communications Technologies, vol 39 Springer, Cham.

[52] Dr Reeja S R, NP Kavya, "A System for Movement Detecting Congestion", INTERNATIONAL JOURNAL OF COMPUTERS \& TECHNOLOGY, Vol.13, No.4. 